\title{
Efecto de Diferentes Cultivos y Prácticas Culturales Sobre la Multiplicación del Nematodo Quiste de la Papa (Globodera spp.) en Bolivia
}

\author{
G. Pacajes ${ }^{1}$ Javier Franco, Raúl Esprella y Gladys Main ${ }^{2}$
}

\begin{abstract}
Resumen
Bajo condiciones del altiplano central de Bolivia (Provincia de Ingavi, La Paz) se realizó un estudio de campo para evaluar diferentes especies cultivadas y prácticas culturales sobre el nematodo quiste de la papa (Globodera spp.) y determinar la factibilidad de su incorporación en una estrategia para el manejo integrado de esta plaga. Se evaluaron tres clones de papa con resistencia parcial a $G$. pallida, el cv. Sani Imilla, susceptible (control sin y con nematicida), el cultivo de haba (Vicia faba), el cultivo de cebada (Hordeum vulgare), suelo en descanso e incorporación de haba como abono verde.
\end{abstract}

De acuerdo a la multiplicación de Globodera spp. ocurrida en los clones evaluados, ésta sufrió un incremento de $30 \%$ en el clon 390154.4 , de $13 \%$ en el G84381.9 y se mantuvo invariable en el clon G85472.20, aunque todos ellos mostraron una menor viabilidad en los nuevos quistes formados. La no reducción de las poblaciones del nematodo en el suelo se debería a la presencia de G. rostochiensis, que no fue afectada por la resistencia a $G$. pallida

En los tratamientos de papa, cebada y haba, se realizaron muestreos de suelo antes de la siembra y a la cosecha, con el objeto de determinar el incremento o disminución de la población del nematodo. Para el abono verde, los muestreos se efectuaron a la siembra, la primera cosecha y luego muestreos mensuales durante la descomposición de la materia verde en el suelo al igual que con el descanso de suelo. La práctica de incorporación de haba como abono verde destacó con una reducción de $41 \%$ en la población del nematodo, seguida por los cultivos de haba, cebada y descanso del suelo $(25,23$ y $24 \%)$, respectivamente. La incorporación del abono verde redujo más notoriamente la población de nematodos durante su etapa de descomposición en el suelo, es decir a partir del segundo mes de su incorporación; posteriormente la población se mantuvo estable. La aplicación del nematicida a la siembra también redujo la

1 Parte de tesis para obtener el título de Ingeniero Agrónomo, La Paz, Bolivia.

2 Fundación PROINPA (Producción e Investigación de Productos Andinos), Casilla 4285, Cochabamba, Bolivia. 
población de nematodos en $12 \%$ y los rendimientos en tubérculos fueron relativamente mayores. Por otro lado el cv. Sani Imilla sin nematicida registró el aumento más significativo de la población, ya que ésta se elevó en $90 \%$, confirmando su susceptibilidad a Globodera spp.

Palabras claves adicionales: $\quad \begin{aligned} & \text { "nematodo quiste de la papa", abonos } \\ & \text { verdes, descanso, rotaciones, clones } \\ & \text { de papa. }\end{aligned}$

\title{
The Effect of Different Crops and Cultural Practices on Multiplication of the Potato Cyst Nematode (Globodera spp.) in Bolivia
}

\begin{abstract}
Summary
Under the Central altiplano boliviano conditions (Prov. Ingavi, La Paz) different crops and cultural practices were evaluated for their effect on Globodera spp. Three potato clones with partía! resistance to Globodera pallida, a susceptible potato cv. Sani Imilla (w/wo nematicide), horse bean crop, barley crop, soil fallow and horse bean green manure incorporation. Globodera spp. multiplied in all 3 clones: nematode population increased by $30 \%$ in clon $390154.4,13 \%$ in G84381.9 and it was invariable in clon G85472.20, although low total cyst viability was observed. The poor performance of clones could be due to the presence of Globodera rostochiensis. Potato, barley and horse-bean treatments were soil sampled at planting and harvesting time in order to establish increase/decrease nematode population density. Horse bean green manure treatment was sampled at planting, first harvest and thereafter at monthly intervals likewise fallow plots. Nematode population density was decreased by $41 \%$ in green manure treated plots while after potato, horse-bean and barley crops nematode population decreased by 25,23 and $25 \%$, respectively. The largest nematode population reduction in green manure plots occurred during the decomposing period, this was one month after green material incorporation and after that nematode population was stable. Nematicide treatment at planting time also affected nematode population by $12 \%$ and tuber yield was higher than non-treated potato plants where nematode population increased by $90 \%$, confirming Globodera spp. susceptibility of potato cv. Sani Imilla.
\end{abstract}


Additional Index words:

potato cyst nematode, green manure, fallow, crop rotation, potato clones.

\section{Introducción}

La papa (Solanum tuberosum) es una planta originaria de la región andina de Sudamérica y constituye el principal cultivo alimenticio de Bolivia y uno de los principales del mundo. Sin embargo, al igual que en otros cultivos, la producción y productividad de éste son limitadas por factores abióticos y bióticos. Entre los primeros están las heladas, sequías, granizo, etc. y dentro de los últimos están los insectos-plaga, hongos, bacterias, virus y nematodos (15).

El nematodo quiste de la papa (Globodera spp.) Ilamado también nematodo dorado por Chitwood et al. (3), al igual que el nematodo del rosario de la papa (Nacobbus aberrans), constituyen severos problemas en ciertas áreas con cultivo de papa, elevando los costos de producción y ocasionando escasez de este tubérculo, tanto en la producción de tubérculos-semillas como de consumo. En general, existe desconocimiento por parte de los productores, de los síntomas, daños y control de este nematodo, lo cual es un factor importante que contribuye a su diseminación y a agudizar el problema. Globodera spp., el nematodo quiste de la papa, produce una reducción de hasta el $50 \%$ en el rendimiento en áreas muy infestadas (5). En Bolivia se estimó que de acuerdo a la densidad en que se encuentre en el suelo, puede ocasionar pérdidas en el rendimiento de hasta el $58 \%$ (7), que alcanzaría una pérdida económica en el valor bruto de la producción de papa a nivel nacional de US\$16'000,000 (9). El ataque de estos nematodos puede también favorecer algunas infecciones en las plantas, como la marchitez bacteriana (Ralstonia solanacearum) y la marchitez por Verticillium $(2,13)$.

La primera evidencia del nematodo quiste de la papa se remonta a finales del siglo XIX en Alemania. Empero, en América Latina, centro de origen del nematodo, el primer reporte de su presencia fue posterior (14). Desde esa fecha hasta el presente, en varios países latinoamericanos, entre ellos Bolivia, se realizan trabajos relacionados al control de este nematodo. En algunos de estos países, el control químico con nematicidas es bastante común, por tener un efecto inmediato que se refleja en mayores rendimientos, más no en reducir las poblaciones de nematodos y poner en riesgo la salud y el medio ambiente. Es así que durante un seminario sobre sistemas de producción en papa, llevado a cabo en Colombia (11), se concluyó que uno de los principales problemas en Bolivia y en los demás países bolivarianos es el uso indiscriminado de plaguicidas y el desconocimiento de otras medidas de control. También se afirma que el 
nematodo quiste constituye en Bolivia uno de los principales problemas, cuyo control requiere prioritariamente el desarrollo y la implementación de un programa de manejo integrado. El Centro Internacional de la Papa (CIP) y el Programa de Investigación de la Papa en Bolivia (PROINPA), han venido señalando la necesidad de estudiar diferentes formas de control del nematodo quiste de la papa, y buscando alternativas integrales para lograr mejores resultados en el afán de disminuir los daños que ocasiona en el cultivo de papa.

Estudios efectuados en Perú (1) y Bolivia (4) sobre el efecto de la práctica tradicional de descanso del suelo sobre las poblaciones de Globodera spp. mostraron que la viabilidad de los quistes de este nematodo disminuye con el tiempo. En el primer caso, después de más de 5 años de descanso, no fue posible detectar quistes en muestras de suelo. Por el contrario, en el segundo caso se determinó que un descanso de 10 años y la rotación de cultivos manejados comunalmente reducía la población del nematodo a niveles casi no detectables.

Por otro lado, Iriarte et al. (12) determinó que con el cultivo de haba se redujo en un $30 \%$ la población inicial de $N$. aberrans y Globodera spp., con una tasa de multiplicación de 0.7 para ambos nematodos. Con respecto al efecto de la forma de aplicación y los niveles de abono verde y estiércol sobre el desarrollo y rendimiento del cultivo de papa, los mejores rendimientos se obtuvieron con la aplicación de abono verde al voleo en niveles de 5 a 10 t/ha, porque en cantidades superiores se observó un efecto fitotóxico. Los mayores rendimientos (26.6 t/ha) se obtuvieron con las combinaciones de abono verde al voleo y estiércol en la proporción de 5-10 y con la aplicación al surco (24.8 t/ha) de la combinación 5-5 que superaron al testigo (19.2 t/ha). Cuando los niveles de 5 y 10 t/ha de abono verde aplicados al voleo 0 al surco se ajustaron a las cantidades equivalentes efectivas que se incorporaron al surco, estas fueron de 2.2 a $4.3 \mathrm{t} / \mathrm{ha}$ y de 5 a $10 \mathrm{t} / \mathrm{ha}$, respectivamente. Estos volúmenes representan una incorporación de 0.15 a $0.3 \mathrm{Kg}$. por metro de surco a un distanciamiento de $0.7 \mathrm{~m}$ entre surcos. Asimismo, la combinación 5-5 de abono verde y estiércol presentó la menor tasa de multiplicación de $N$. aberrans y $G$. pallida y el mayor ingreso económico al agricultor, que con el sistema haba-papa obtuvo US\$ 5.9 por cada dólar invertido.

Con la finalidad de establecer las bases para un plan de manejo integrado de Globodera spp., en el presente estudio se evaluaron y compararon algunas alternativas de control -a las que Franco, González y Matos (8), denominan componentes- de Globodera spp. y cuyos resultados sean empleados en la implementación de su manejo integrado. Por lo anteriormente expuesto, en este estudio se evaluó tanto el efecto de diferentes especies cultivadas y prácticas culturales sobre Globodera spp., como el comportamiento de ciertos cultivares y clones de papa ante la presencia de este nematodo. 


\section{Materiales y Métodos}

Este estudio se desarrolló en campo y bajo condiciones de secano, en una parcela ubicada en la provincia Ingavi del departamento de La Paz, Altiplano Central de Bolivia, a una altitud de 3798 msnm La precipitación promedio anual fue de $582 \mathrm{~mm}$ y la temperatura promedio anual de 8.01 ${ }^{\circ} \mathrm{C}$.

La parcela experimental fue seleccionada por su infestación natural con Globodera spp. y la instalación se realizó usando un diseño de bloques completos al azar, con 9 tratamientos y 4 repeticiones. Dentro de cada tratamiento se identificaron cuatro unidades experimentales elegidas al azar, cada una con una área de $4 \mathrm{~m}^{2}(2 \times 2 \mathrm{~m})$. Los clones G84381.9, 390159.4 y G85472.20 se incluyeron por ser considerados por el Centro Internacional de la Papa (CIP), como parcialmente resistentes a G. pallida. Los diversos tratamientos se indican en la Tabla 1.

Tabla 1. Tratamientos estudiados bajo condiciones de campo (La Paz, Bolivia).

Código

Tratamientos

\begin{tabular}{ll}
\hline PR1 & Clon G 84381.9 (Resistencia parcial a G. pallida) \\
PR2 & Clon G 390159.4 (Resistencia parcial a G. pallida) \\
PR3 & Clon G 85472.20 (Resistencia parcial a G. pallida) \\
LNI & Haba (No hospedante) \\
PS & Papa, var. Sani Imilla (Susceptible a Globodera spp.) \\
PS+N & Papa, var. Sani Imilla (+nematicida Carbodan 48 FW) \\
C & Cebada (No hospedante) \\
L & Abono verde de haba \\
D & Descanso
\end{tabular}

La siembra de los tratamientos con los cultivos de papa y haba se realizó el 17 de noviembre de 1995. La siembra de cebada se efectuó el 29 de noviembre de 1995. En el tratamiento correspondiente al descanso del suelo, se procedió simplemente al marcado y delimitación de las parcelas.

La cosecha de los tratamientos con cultivos de papa se efectuó el 26 de abril de 1996. En cuanto al tratamiento con cultivo de haba, se realizaron tres cosechas, la última cosecha fue el 16 de mayo de 1996. La cebada fue cosechada el 14 de marzo de 1996 en pleno estado lechoso de las espigas.

En el tratamiento con abono verde, se realizó una primera cosecha de vainas verdes, y luego la siega y volteo del follaje el 28 de abril de 1996, para ser incorporada al suelo como abono verde, el 29 de abril de 1996.

De manera previa a la siembra y también después de la cosecha de los cultivos de papa, haba y cebada, de cada unidad experimental se tomó una muestra de suelo (1 kilogramo) a una profundidad de 15 a $20 \mathrm{~cm}$, 
correspondiendo éstas a las poblaciones iniciales $(\mathrm{Pi})$ y finales (Pf), respectivamente. En el tratamiento abono verde, las muestras de suelo se tomaron a la siembra (noviembre, 1995), a la primera cosecha (abril, 1996) y se continuaron tomando durante el proceso de descomposición de la materia verde a intervalos de un mes, hasta la siguiente época de siembra (octubre, 1996). En el tratamiento descanso del suelo, se tomaron muestras al momento de la siembra de los otros tratamientos (noviembre, 1995) y la cosecha del cultivo de las papas (mayo, 1996), y una última muestra fue tomada al inicio de la siguiente campaña agrícola (octubre, 1996), para efectos de comparación con el tratamiento con abono verde.

En laboratorio y para cada muestra de suelo correspondientes a las Pi y Pf, se determinó el número de quistes por $100 \mathrm{~g}$ de suelo, el número de juveniles y huevos por quiste (Viabilidad total: VT), para posteriormente estimar el nivel de infestación por el número de juveniles y huevos por gramo de suelo de acuerdo a la siguiente fórmula (10):

\section{$\mathrm{N}^{\mathrm{a}}$ de juveniles y huevos $/ \mathrm{g}$ de suelo $=\mathrm{VT} \times$ Número de quistes $/ 100 \mathrm{~g}$ de suelo}

Estos valores de la población inicial (a la siembra) o final (a la cosecha, en el caso de los cultivos de papa, haba y cebada) en los diversos tratamientos, fueron utilizados para establecer la tasa de multiplicación de nematodos (TMN) mediante el siguiente cociente:

$$
\text { TMN }=\frac{\text { Población final de nematodos }(\mathrm{Pf})}{\text { Población inicial de nematodos }(\mathrm{Pi})}
$$

Si TMN > 1, se asume que la población de nematodos incrementa

$\mathrm{Si} \mathrm{TMN} \leq 1$, se asume que la población de nematodos decrece

La extracción de quistes de las muestras de suelo, se efectuó por el método de Fenwick y tratamiento en compuestos orgánicos (gasolina).

En el tratamiento con abono verde, se determinó la población de nematodos a la siembra (noviembre, 1995), al momento de la primera cosecha e incorporación de materia verde (abril, 1996) y durante el proceso de descomposición de la materia verde en el suelo a intervalos de un mes hasta la siguiente siembra (octubre, 1996). En el tratamiento descanso del suelo se determinó las poblaciones al momento de la siembra, la cosecha de los cultivos de papa y al inicio de la siguiente siembra (octubre, 1996). Asimismo, en los clones y cultivares de papa se evaluó además el peso de raíz, invasión de Globodera spp. a las raíces a los 35 días después de la siembra y los rendimientos de cada cultivo. 


\section{Resultados y Discusión}

La parcela experimental presentó una distribución heterogénea de la población de Globodera spp. Asimismo, mediante observaciones de la región Terminal de los quistes, se detectó la presencia de las dos especies, G. pallida y $G$. rostochiensis. La Tabla 2 muestra los promedios de población inicial y población final de los tratamientos.

Tabla 2. $\quad$ Promedios de población inicial (Pi) y población final (Pf) de Globodera spp.

\begin{tabular}{|c|c|c|c|c|c|c|}
\hline \multirow[t]{2}{*}{ Tratamientos } & \multicolumn{3}{|c|}{ Población inicial (Pi) } & \multicolumn{3}{|c|}{ Población final (Pf) } \\
\hline & $\begin{array}{l}\text { Quiste/100g } \\
\text { suelo }\end{array}$ & $\begin{array}{c}\mathrm{J}+\mathrm{H} \text { v/quiste } \\
\text { (VT) }\end{array}$ & $\begin{array}{c}\mathrm{J}+\mathrm{Hv} / \mathrm{g} \\
\text { suelo }\end{array}$ & $\begin{array}{c}\text { Quiste } / 100 \mathrm{~g} \\
\text { suelo }\end{array}$ & $\begin{array}{c}\mathrm{J}+\mathrm{Hv} / \text { quiste } \\
\text { (VT) }\end{array}$ & $\begin{array}{l}\mathrm{J}+\mathrm{Hv} / \mathrm{g} \\
\text { suelo }\end{array}$ \\
\hline Clon G84381.9 & 67.05 & 103.33 & 69.32 & 80.68 & 97.00 & 78.21 \\
\hline Clon 390159.4 & 72.23 & 152.92 & 111.50 & 114.36 & 117.92 & 133.77 \\
\hline Clon G85472.20 & 89.77 & 157.33 & 142.18 & 101.36 & 139.33 & 142.39 \\
\hline Haba & 108.55 & 168.58 & 181.89 & 94.70 & 143.33 & 133.56 \\
\hline Papa (var.Sani I.) & 99.20 & 141.00 & 141.48 & 191.42 & 136.17 & 266.07 \\
\hline Papa+nematicida & 86.27 & 125.17 & 115.86 & 63.32 & 144.58 & 97.27 \\
\hline Cebada & 88.55 & 147.92 & 131.35 & 82.04 & 123.92 & 103.70 \\
\hline Abono verde $\left({ }^{*}\right)$ & 87.09 & 193.92 & 165.27 & 76.65 & 130.84 & 97.91 \\
\hline Descanso $\left({ }^{*}\right)$ & 101.56 & 178.17 & 192.69 & 84.90 & 152.50 & 138.26 \\
\hline
\end{tabular}

$\left({ }^{*}\right)$ En los tratamientos abono verde y descanso, la Pf corresponde a octubre de 1996.

\section{Tasa de multiplicación de quistes (TMQ)}

En el cultivar Sani Imilla se expresó la mayor TMQ, confirmando Sani Imilla su susceptibilidad al ataque de los nematodos quistes. En los clones 390159.4, G84381.9 y G85472.20 también se obtuvieron valores de TMQ que sobrepasaron la unidad, sin embargo, esto es justificable por la presencia de G. rostochiensis en el suelo del ensayo, que se desarrollaron y multiplicaron libremente ya que los clones expresan resistencia genética sólo a $G$. pallida. 


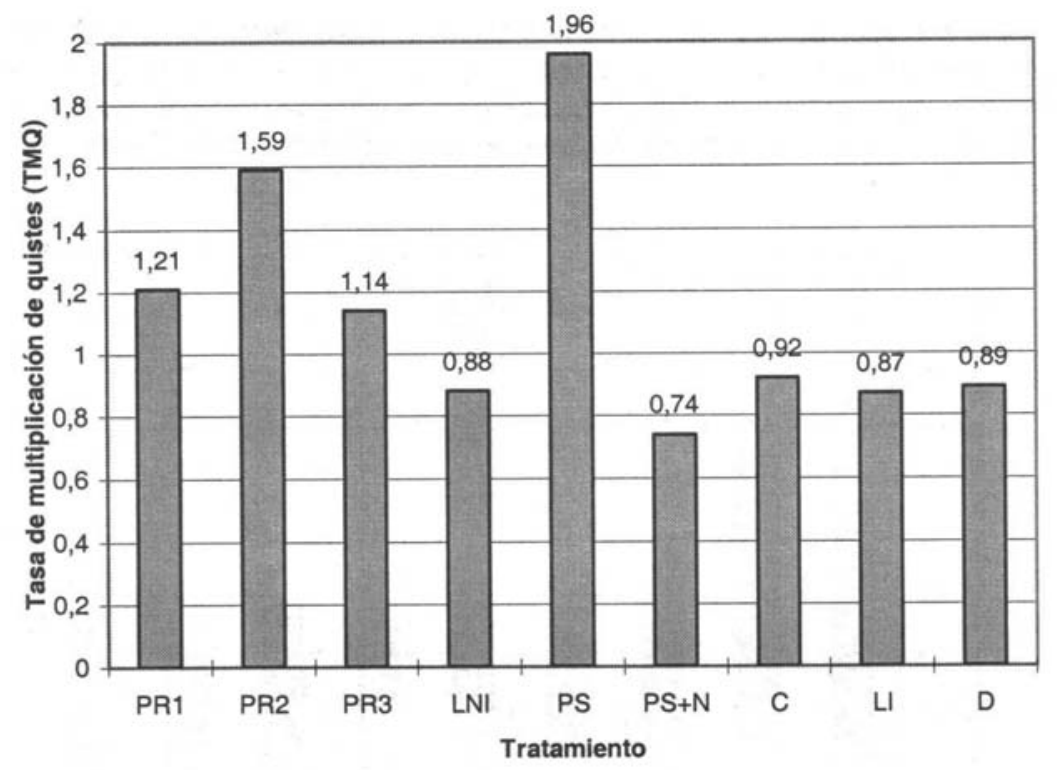

Figura 1. Efecto de diferentes cultivos y prácticas culturales sobre la TMQ (PR1; PR2 y PR3: clones de papa; LNI: haba; PS: papa; $P S+N$ : papa+nematicida; C: cebada; LI: Abono verde; $D:$ descanso).

En el tratamiento con control químico se presentó una tasa de multiplicación de quistes (TMQ) significativamente inferior a las demás, lo cual explica que el producto empleado sí fue efectivo con relación a la formación de nuevos quistes, pero en la dosis aplicada que fue relativamente alta. Sin embargo, en la Tabla 2 se observa que, después de la cosecha, la VT incrementó en el tratamiento con control químico, esto pudo deberse a que los nematodos que sobrevivieron al efecto nematicida, tuvieron un hábitat favorable para desarrollarse y multiplicarse.

La población de quistes decreció, después del cultivo de cebada, haba, barbecho y la práctica cultural de abono verde de haba. La ausencia de hospedantes eficientes, la humedad, temperatura y ciertos microorganismos de suelo habrían influido en la destrucción de los quistes más longevos.

\section{Tasa de multiplicación de nematodos (TMN)}

La mayor TMN en huevos y juveniles por gramo de suelo se expresó después del cultivo de Sani Imilla, confirmando su susceptibilidad a Globodera spp. 
En cambio, el control químico logró disminuir la población de nematodos en el suelo, al eliminar una gran proporción de los juveniles emergidos y brindar al cultivo una protección el tiempo suficiente como para impedir que los nematodos sobrevivientes alcancen una TMN más alta.

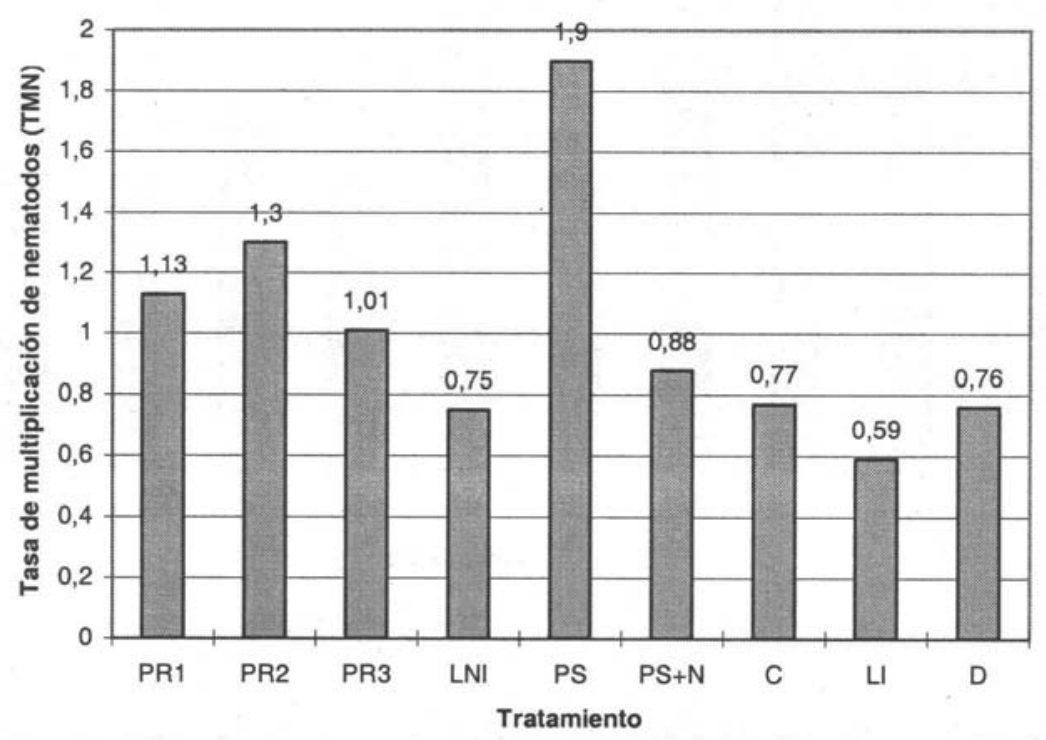

Figura 2. Efecto de diferentes cultivos y prácticas culturales sobre la TMN (PR1; PR2 y PR3: clones de papa; LNI: haba; PS: papa; $P S+N$ : papa+nematicida; C: cebada; U: Abono verde; D: descanso).

En el grupo de los clones también se dio cierto incremento en la población de nematodos, sobretodo en el clon 390159.4. El desarrollo y multiplicación de $G$. rostochiensis habría influido en el incremento de la población de nematodos después del cultivo de los tres clones, a diferencia de G. pallida, al cual son resistentes los clones.

Después del cultivo de cebada, haba, barbecho y abono verde de haba, disminuyó la población de nematodos en el suelo, la disminución fue notable en el tratamiento con abono verde. La ausencia de hospedantes eficientes, la acción de ciertos exudados radiculares, la humedad, temperatura y microorganismos del suelo habrían influido para esta disminución de la población de nematodos en el suelo lo que se conoce como mortandad natural. En el siguiente punto se hace un análisis sobre la acción del abono verde de haba en la disminución de nematodos en el suelo, en comparación con el descanso del suelo.

\section{Efecto del abono verde en la población del nematodo quiste en relación con el descanso del suelo}


En el tratamiento abono verde, se determinaron las poblaciones de nematodos en las etapas de siembra (noviembre, 1995), primera cosecha e incorporación de materia verde (abril, 1996) y, posteriormente, cada mes hasta el inicio de la siguiente gestión agrícola (octubre, 1996). En el tratamiento descanso del suelo la determinación de población de nematodos correspondieron a la siembra de papa (noviembre, 1995), a la cosecha (abril, 1996) y una última evaluación en octubre de 1996. La Figura 3 muestra que en el caso del abono verde, la población de quistes disminuyó desde la siembra del haba (noviembre, 1995) a la primera cosecha (abril, 1996) en un 7 por ciento. La disminución de la población de quistes, desde la incorporación (abril, 1996) hasta el último mes muestreado (octubre, 1996), se dio en una proporción del 5 por ciento. En total, desde la siembra hasta el último muestreo, se evidenció una reducción de $13 \%$.

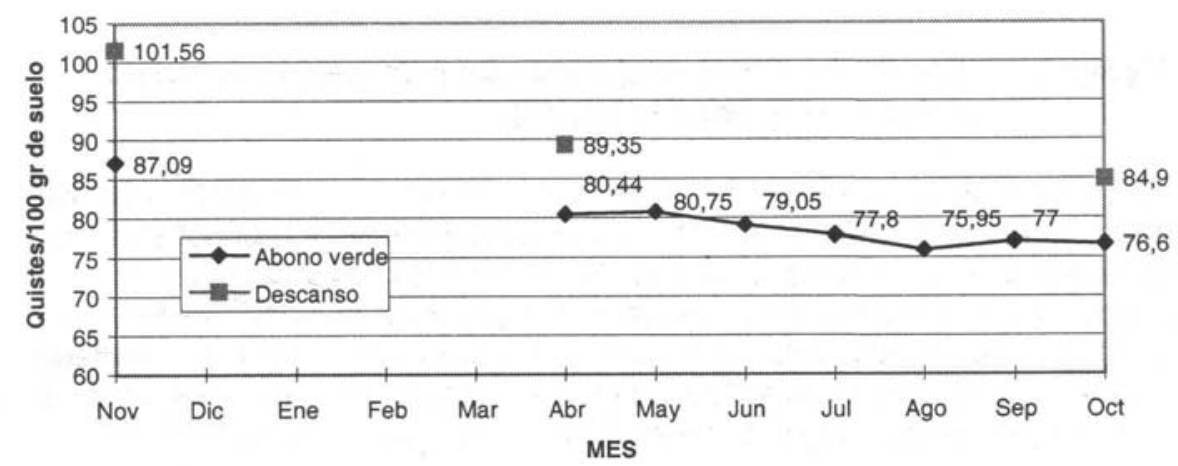

Figura 3. Efecto de abono verde de haba y descanso del suelo sobre la población de quistes de Globodera spp.

Para el tratamiento descanso del suelo, desde noviembre de 1995 a abril de 1996, la población de quistes disminuyó en 12 por ciento y de abril a octubre de 1996, la reducción fue en 5 por ciento. La reducción total desde noviembre de 1995 a octubre de 1996 fue en una proporción aproximada del 11 por ciento. Este análisis permite deducir que, comparando los efectos del tratamiento abono verde y descanso del suelo, se evidenció que ambos tuvieron un efecto prácticamente similar en la disminución de la densidad de quistes, que sería atribuible a una posible destrucción o descomposición natural de los quistes más antiguos por acción de ciertos factores que favorecieron una mayor actividad microbiana en el suelo.

Asimismo, de acuerdo a la Figura 4, Globodera spp. presentó fluctuaciones marcadas en su viabilidad total (VT) por efecto del abono verde, principalmente después de la incorporación de la materia verde al suelo. El proceso físico-químico de la descomposición del abono verde 
habría sido el factor preponderante para una mayor reducción del número de huevos y juveniles dentro de los quistes (32.5\%). En el descanso del suelo, aún cuando también se observa disminución de la VT, ésta fue en una tasa relativamente menor $(14.4 \%)$,con una diferencia del $18.1 \%$ a favor de la incorporación del abono verde.

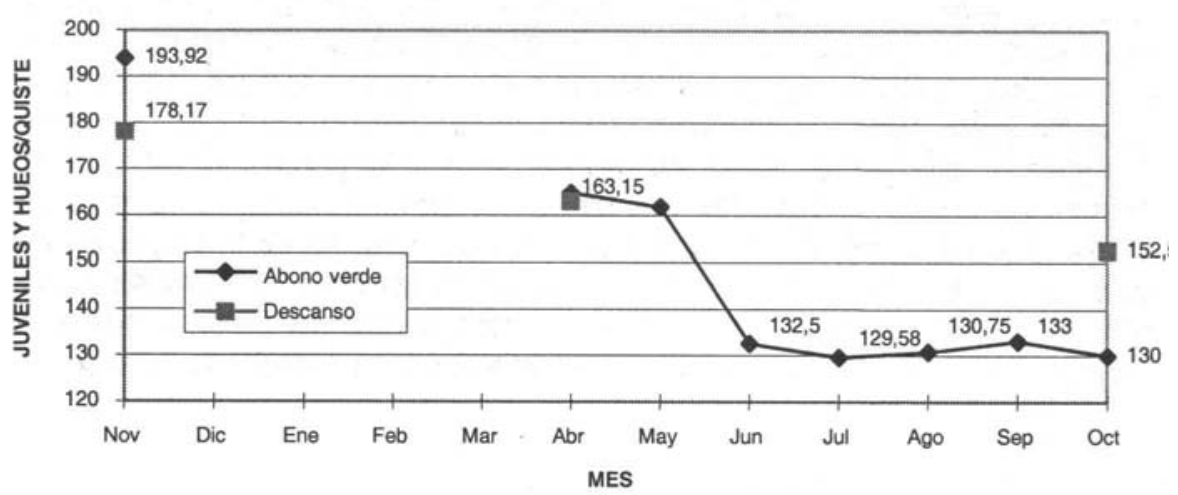

Figura 4. Efecto de abono verde de haba y descanso del suelo sobre la viabilidad total $(V T)$.

Así mismo al compararse los niveles de infestación de estos mismos tratamientos (Figura 5), se evidencia que en el tratamiento abono verde, de la siembra a la primera cosecha, hubo reducción de la población de nematodos en 20 por ciento y desde la incorporación de abono verde hasta el último mes muestreado, la reducción fue de $25 \%$, dando un resultado global de 41 por ciento de reducción en la población de nematodos desde la siembra (noviembre, 1995) hasta el último muestreo (octubre, 1996).

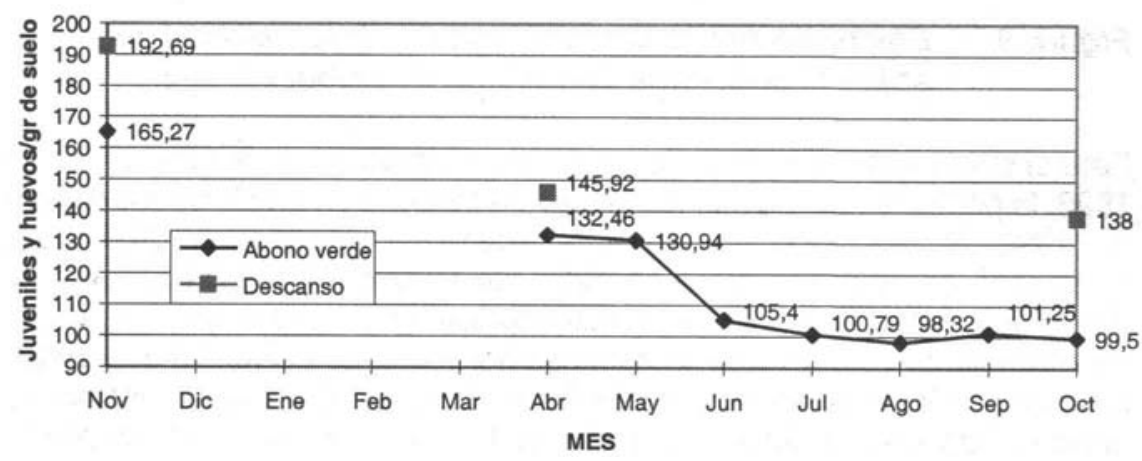

Figura 5. Efecto de abono verde de haba y descanso del suelo sobre la población de nematodos de Globodera spp. 
Comparando con el efecto del descanso del suelo (28\% de reducción total, de noviembre de 1995 a octubre de 1996), la acción del abono verde en la disminución de nematodos fue relativamente mayor. El proceso físico-químico por el que atravesó la descomposición de la materia verde de haba, fue determinante en la reducción de la población de nematodos en el suelo, afectando principalmente la viabilidad total de los quistes (VT).

\section{Comportamiento de clones de papa a Globodera spp.}

Para esta evaluación se consideró el peso de raíz en fresco, el número de estados juveniles por gramo de raíz, el número de estados juveniles por raíz y el rendimiento de Sani Imilla y los diferentes clones de papa (Tabla 3).

Tabla 3. Peso de raíz en fresco, número de estados juveniles por gramo de raíz, número de estados juveniles por raíz y rendimiento de los clones y cultivar de papa.

\begin{tabular}{lccc}
\hline \multicolumn{1}{c}{ Tratamientos } & $\begin{array}{c}\text { Peso de } \\
\text { raíz en } \\
\text { fresco } \mathbf{( g )}\end{array}$ & $\begin{array}{c}\mathbf{N}^{\circ} \text { de estados } \\
\text { juveniles/g raíz }\end{array}$ & $\begin{array}{c}\mathbf{N}^{\circ} \text { de estados } \\
\text { juveniles en } \\
\text { raíces }\end{array}$ \\
\hline Clon G84381.9 & 32.94 & 13.50 & 444.63 \\
Clon 390159.4 & 28.29 & 15.25 & 432.73 \\
Clon G85472.20 & 27.22 & 15.50 & 430.43 \\
Sani Imilla & 28.25 & 16.25 & 462.08 \\
Sani Imilla más nematicida & 34.57 & 7.50 & 260.65 \\
\hline
\end{tabular}

De acuerdo a los resultados obtenidos, el cultivar Sani Imilla con tratamiento químico presentó el mayor peso de raíces. El control químico con Carbodan 48FW, al eliminar los nematodos y otros insectos en la etapa inicial del cultivo, contribuyó a que las raíces tuvieran un mejor desarrollo en comparación con el cultivo de Sani Imilla sin tratamiento químico, aunque el clon G84381.9 también alcanzó un buen nivel de desarrollo que podría atribuirse en parte a sus características genéticas. Los promedios de peso de raíz de los demás cultivares presentaron pocas variaciones.

La menor invasión de Globodera spp. por gramo de raíz se dio en el cultivar Sani Imilla tratada con nematicida que redujo el número de juveniles en 54 $\%$ con respecto al cultivar Sani Imilla sin aplicación de nematicida, que registró el promedio más elevado. Las respectivas TMN siguieron el mismo patrón, es decir la alta dosis de nematicida empleada redujo la población en $12 \%$, en contraste con S. Imilla sin nematicida que la incrementó en $90 \%$. Los otros tres clones de papa registraron valores promedios del número de estados juveniles en raíces, relativamente iguales y escasamente inferiores al valor más alto, correspondiente a Sani Imilla. Sin embargo cuando se comparan las respectivas TMN (Figura 2), se observa que en el clon G 85472.20 ésta fue casi nula (1.01) en relación a los otros dos, en los que ocurrió mayores multiplicaciones (13 y $30 \%$ ), pero que en todo caso fueron 
menores a la ocurrida en Sani Imilla, indicando un cierto grado de resistencia a Globodera spp.

Comparando los rendimiento de este grupo de tratamientos (Tabla 3), el cultivar Sani Imilla tratado con nematicida, presentó un rendimiento substancialmente superior al resto de los demás tratamientos y un incremento de $30.2 \%$ sobre el rendimiento del cultivar Sani Imilla sin aplicación de nematicida, este incremento sería en respuesta al efecto inicial del producto nematicida. Los rendimientos del grupo de los clones de papa, fueron menores al de Sani Imilla, posiblemente por falta de adaptación, no mostrando diferencias substanciales, aunque el mejor rendimiento correspondió al clon 390159.4.

En general, las fluctuaciones de rendimiento en todos los cultivares y clones de papa, no pueden ser atribuibles solamente a la incidencia de los nematodos, ya que también han influido características agronómicas inherentes a cada clon o cultivar.

\section{Referencias Bibliográficas}

1. Blanco, O. 1994. Efecto del descanso del suelo sobre el número y la viabilidad de quistes de Globodera spp. en la papa. In: Dinámicas del descanso de la tierra en los Andes. Eds.D. Hervé, D. Genin y G. Riviere, IBTA-ORSTOM, La Paz, Bolivia: 171-174.

2. Centro Internacional de la Papa. 1996. Principales enfermedades, nematodos e insectos de la papa. Asociación Editorial Stella. LimaPerú. $72 \mathrm{p}$.

3. Chitwood, B.G., R.L. Clement, R. Morgan, R. Tank. 1942. Heterodera rostochiensis, the golden nematode in New York State. Plant Disease Repórter 26: 390-391.

4. Esprella R., D. Hervé, J. Franco. 1994. Control del Nematodo Quiste de la papa (Globodera pallida) por el descanso controlado comunalmente: Altiplano Central Boliviano. In: Dinámicas del descanso de la tierra en los Andes. Eds.D. Hervé, D. Genin y G. Riviere, IBTA-ORSTOM, La Paz, Bolivia: 175-183.

5. Foot, M., F. Wood, J. Currie. 1980. Potato cyst nematode in New Zealand. Entomology División (Pukekohe) N 1: 3-21.

6. Franco, J. 1986. Nematodos del quiste de la papa. Boletín de Información Técnica, Centro Internacional de la Papa. Lima, Perú. 19 P-

7. Franco, J. 1994. Problemas de nematodos en la producción de papa en climas templados en la región andina. Nematrópica 24:179-195.

8. Franco, J., A. Gonzales, A. Matos. 1993. Manejo integrado del nematodo quiste de la papa. Centro Internacional de la Papa (CIP), Programa de Investigación de la Papa (PROINPA), Cochabamba, Bolivia. $172 \mathrm{p}$. 
9. Franco, J., J Ramos, R. Oros, G. Main, N. Ortuño. 1998/1999. Pérdidas económicas causadas por Nacobbus aberrans y Globodera spp. en el cultivo de la papa en Bolivia. Revista Latinoamericana de la Papa 11: 40-66.

10. Gonzáles, A., J. Franco. 1993. Técnicas y Métodos para el nematodo quiste de la Papa. Centro Internacional de la Papa (CIP), Programa de Investigación de la Papa (PROINPA), Cochabamba, Bolivia. $100 \mathrm{p}$.

11. Instituto Interamericano de Cooperación Para la Agricultura. 1987. Seminario sobre sistemas de producción en papa. Noviembre, 1987. Pasto, Colombia.

12. Iriarte, L, J. Franco, N. Ortuño. 1998/1999. Efecto de Abonos Orgánicos sobre las Poblaciones de Nematodos y la Producción de la Papa. Revista Latinoamericana de la Papa 11:149-163.

13. National Academy of Sciences. 1977. Control de plagas de plantas y animales. Control de nematodos parásitos de plantas. Volumen 4. Editorial Limusa, México.219 p.

14. Wille, J.E., C. Bazán de Segura. 1952. La Anguillula Dorada, Heterodera rostochiensis, una plaga del cultivo de las papas recién descubierta en el Perú. Boletín № 48, Estación Experimental Agrícola La Molina, Lima, Perú.17 p.

15. Zeballos, H. 1997. Aspectos económicos de la producción de papa en Bolivia. Informeal Centro Internacional de la Papa (CIP) y el Programa de Investigación de la Papa (PROINPA). 209 p. 